\title{
Efficacy of Iodine-125 Seed Implantation in Locoregionally Recurrent and Unresectable Breast Cancer: a Retrospective Study
}

\author{
Ying-hua Yu ${ }^{1}$ (D) Chang-yuan Wei ${ }^{1} \cdot$ Qing-hong Qin ${ }^{1} \cdot$ Qin-guo Mo $^{1} \cdot$ Zhen Huang $^{1}$. \\ Bin Lian ${ }^{1}$
}

Received: 12 June 2017 / Accepted: 27 October 2017 / Published online: 7 November 2017

(C) The Author(s) 2017. This article is an open access publication

\begin{abstract}
The management of locoregionally recurrent and unresectable breast cancer is a therapeutic challenge. This retrospective study aimed to assess the efficacy of ${ }^{125}$ I seed implantation brachytherapy as a palliative management in locoregionally recurrent breast cancer. We analyzed 36 locoregionally recurrent and unresectable breast cancers in our hospital between 2012 and 2016. All patients were treated with CT-guided ${ }^{125}$ I seed permanent implantation. The dose distribution of ${ }^{125}$ I seeds was calculated using a computerized treatment planning system. Complete response, partial response, stable disease, and local tumor control rates were calculated. Long-term efficacy was assessed based on survival rates ranging from 1 to 4 years. The follow-up period ranged from 6 to 53 months. The median local control was 28 months (95\% CI: 16.2-39.8 months). The percentage of patients who showed 6-month, 1-year, 2-year, and 3-year local control was
\end{abstract}

Chang-yuan Wei

weicy63@aliyun.com

Ying-hua Yu

carolyu111@139.com

Qing-hong Qin

86822544@qq.com

Qin-guo Mo

qgmo135@263.net

Zhen Huang

48577880@qq.com

Bin Lian

116846715@qq.com

1 Departmant of Breast Surgery of Affiliated Tumor Hospital of Guangxi Medical University, NO. 71, He Di Lu,

Nanning, Guangxi 530021, People's Republic of China
$97.2 \%, 77.8 \%, 52.8 \%$, and $33.3 \%$, respectively. Median survival time for all patients was 48 months (95\% CI: 40.9 55.1 months); 1-year, 2-year, 3-year, and 4-year survival rates were $97.2 \%, 80.6 \%, 63.9 \%$, and $46.5 \%$, respectively. Pain relief response rate was $88.9 \%$. No serious complications were detected during the follow-up period. The results of this study demonstrate that ${ }^{125}$ I seed implantation could be considered a feasible and promising minimally invasive therapy for locoregionally recurrent and unresectable breast carcinoma.

Keywords Iodine-125 seed implantation · Brachytherapy . Locoregionally $\cdot$ Unresectable $\cdot$ Breast cancer

$\begin{array}{ll}\text { Abbreviations } \\ \text { CI } & \text { Confidence interval } \\ \text { EBRT } & \text { External beam radiation therapy } \\ \text { GTV } & \text { Gross tumor volume } \\ \text { LRBC } & \text { Locoregionally recurrent breast cancer } \\ \text { MPD } & \text { Minimal peripheral dose } \\ \text { NRS } & \text { Numerical Rating Scale } \\ \text { PTV } & \text { Planning treatment volume } \\ \text { ReRT } & \text { Reirradiation } \\ \text { RT } & \text { Radiation therapy } \\ \text { TPS } & \text { Treatment planning system }\end{array}$

\section{Introduction}

The number of patients experiencing locoregionally recurrent breast cancer (LRBC) has been increasing steadily over the past decade. After mastectomy, $2 \%$ to $31 \%$ of patients suffer from a second local recurrence [1]. Even after adjuvant radiation therapy (RT), the rate of locoregional recurrences remains around $5 \%$ to $15 \%[2,3]$. Almost $80 \%$ of these 
recurrences appear usually within the first 5 years from the primary treatment [4], and approximately $10 \%$ of them are represented by inoperable breast cancer. Therefore, a promising palliative therapy should be considered for these patients.

The management of LRBC is a therapeutic challenge. The most common therapies involve surgery, external beam RT (EBRT), chemotherapy, or hormonal interventions [5]. However, these treatments still have their limitations. Some of the locally recurrent breast tumors cannot be completely removed. Chemotherapy failed to achieve therapeutic benefits, and some recurrent breast cancer patients are not responding to endocrine therapy, as shown by a systematic review of randomized trials [6]. Excessive external beam radiotherapy could increase the probability of both acute and late toxicities, because of the risk of exceeding the radiation tolerance limits. Therefore, another palliative therapy should be considered.

Recently, image-guided iodine-125 $\left({ }^{125} \mathrm{I}\right)$ seed brachytherapy has received more and more attention, several studies reporting that ${ }^{125}$ I seed brachytherapy provided promising results for the local control of solid tumors such as head and neck cancers, prostatic malignancies, and many other malignant tumors [7-11]. In these studies, ${ }^{125}$ I seed implantation was capable of delivering a sufficient dose of radiation to the tumor mass, causing less damage to adjacent normal tissues.

Although ${ }^{125}$ I radioactive seeds were studied and applied in prostate cancer and other tumor types, they have been relatively neglected in LRBC treatment, being used at present for the localization of non-palpable breast tumors, or to mark the tumor bed for neo-adjuvant chemotherapy or breastconserving surgery $[12,13]$. Up to now, no reports are available investigating ${ }^{125}$ I seed implant brachytherapy as a multimodality therapy of LRBC.

Hence, we performed this retrospective study to investigate the efficacy of ${ }^{125}$ I seed implantation in LRBC and to determine whether this therapy could be beneficial as a palliative method in this recurrent cancer.

\section{Materials and Methods}

\section{Patients}

Thirty-six patients, 36-86 years old (median age 46 years), with locally advanced breast cancer were enrolled in this retrospective study conducted from January 2012 to June 2016. During this period, the patients underwent computed tomography (CT)-guided ${ }^{125}$ I seed implantations at the Breast Center of Affiliated Cancer Hospital of Guangxi Medical University, Guangxi, China. The inclusion criteria were the following: patients enrolled in the study had a history of surgery, adjuvant chemotherapy, or hormonal interventions, with or without EBRT; unresectable and locally recurrent breast lesions were confirmed by histopathological or cytopathological examination; ultrasound or CT scan indicated a solid mass or nodule in the local area. These patients were not suitable for salvage surgery alone after being reviewed by surgeons. Among these patients, 14 had chest wall metastasis, 10 had axillary metastasis (which caused edema of the upper extremity), 8 had supraclavicular metastasis, and 4 had metastasis in other sites. The characteristics of these patients are listed in Table 1.Written informed consent was obtained from all patients, and the study was approved by the institutional ethics committee of the Affiliated Tumor Hospital of Guangxi Medical University.

\section{Treatment Process}

Three days prior to ${ }^{125} \mathrm{I}$ seed implantation, all patients underwent a CT scan. According to the CT image, the total volume of each tumor was calculated using a treatment planning system (TPS) [14]. The dose of ${ }^{125}$ I seed implantation was prescribed as the minimal peripheral dose (MPD), which encompassed the planning treatment volume (PTV). PTV included the entire gross tumor volume (GTV) and $0.5-1.0 \mathrm{~cm}$ margins that were outlined by the radiation oncologist on each CT transverse image.

Table 1 Patient charateristics $(n=36)$

\begin{tabular}{|c|c|c|}
\hline Characteristic & Value & Percentage $(\%)$ \\
\hline Median age (range) & $46(36-86)$ & \\
\hline \multicolumn{3}{|l|}{ Primary tumor stage(n) } \\
\hline stage I & 2 & 5.5 \\
\hline stage II & 5 & 13.9 \\
\hline stage III & 19 & 52.8 \\
\hline stage IV & 8 & 22.2 \\
\hline Unclear & 2 & 5.6 \\
\hline \multicolumn{3}{|l|}{ Tumor site(n) } \\
\hline chest wall & 14 & 38.9 \\
\hline axillary & 10 & 27.8 \\
\hline supraclavicular & 8 & 22.2 \\
\hline other site & 4 & 11.1 \\
\hline \multicolumn{3}{|l|}{ KPS(n) } \\
\hline 50 & 11 & 30.5 \\
\hline 60 & 9 & 25 \\
\hline 70 & 11 & 30.6 \\
\hline$\geq 80$ & 5 & 13.9 \\
\hline Previous surgery & 36 & 100 \\
\hline Previous chemotherapy & 34 & 94.4 \\
\hline Previous radiotherapy & 34 & 94.4 \\
\hline One & 3 & 8.3 \\
\hline Two & 26 & 72.2 \\
\hline Three & 5 & 13.9 \\
\hline Four & 2 & 5.6 \\
\hline
\end{tabular}

KPS = Karnofsky Performance Status score 
The expected number of implanted seeds was calculated according to the modified level formula [15]. The length of ${ }^{125}$ I seeds (Model 6711; Beijing Atom and High Technique Industries Inc., Beijing) was $4.5 \mathrm{~mm}$, and the diameter was less than $1 \mathrm{~mm}$. The properties of ${ }^{125} \mathrm{I}$ seeds were the following: low X-ray energy level of $27.4-31.4 \mathrm{KeV}$, a half-life ( $\mathrm{t}$ $1 / 2$ ) of 59.6 days, and a half-value of tissue penetration of approximately $1.7 \mathrm{~cm}$. These properties gave a sharp dropoff dose and allowed a safe handling. Specific equipment, such as 18-G implantation needles and turntable implantation gun (XinKe Pharma-ceutical Ltd., Shanghai, China), was used. The implant parameters are listed in Table 2.

The therapeutic intervention was performed in the CT room. All patients underwent local anesthesia before ${ }^{125}$ I seed implantation. The procedure time was approximately $30-160 \mathrm{~min}$. The operation procedure was as follows: under CT supervision, 18gauge interstitial needles were inserted through the skin, into the tumor lesion, within the PTV. Most of the needles were placed in a parallel array $1.0-1.5 \mathrm{~cm}$ apart and extended at least $0.5-1 \mathrm{~cm}$ beyond the GTV. Next, the ${ }^{125}$ I seeds were implanted using a Mick applicator. Two to six seeds per needle were loaded and released every $0.5-1 \mathrm{~cm}$ apart upon needle withdrawal. During the procedure, neural structures and large vascular structures were carefully avoided. The median number of ${ }^{125}$ I seeds used in our study was 36 (range 8-101). Median procedure time was 42 min (range 26-93 min). Puncture site was bandaged and compressed to achieve hemostasis after the procedure. Patients remained under observation at the hospital for 1 or 2 full days.

\section{Post ${ }^{125}$ I Seed Implantation}

After seed implantation, 28 of the 36 patients received 4-6 cycles of chemotherapy, and eight patients received endocrine therapy. Chemotherapy and endocrine therapy were performed according to the latest guidelines of the National Comprehensive Cancer Network (NCCN).

\section{Clinical Effect Evaluation}

All 36 patients started the follow-up phase immediately after ${ }^{125}$ I brachytherapy. The median follow-up was 45.9 months

Table 2 Implant parameters

\begin{tabular}{lll}
\hline Characteristic & Median & Range \\
\hline Volume implanted(cm3) & 39 & $3.6-143.2$ \\
MPD (Gy) & 110 & $90-140$ \\
Activity/seed (mCi) & 0.6 & $0.4-0.7$ \\
Numbers of seeds & 36 & $8-101$ \\
Total activity (mCi) & 22.9 & $2.6-86.4$ \\
Dose rate $(\mathrm{Gy} / \mathrm{h})$ & 0.07 & $0.05-0.09$ \\
\hline
\end{tabular}

(range 14.9-53 months). Clinical examination, blood sampling, ultrasound, and CT examination at 1 month, 2 months, and every 3 months post-intervention in the first year were performed. Next, the above medical check-up was performed every 6 months. One case was lost during the follow-up period, after 33.6 months.

Tumor response was assessed according to World Health Organization (WHO) criteria [16]. Complete response (CR) was defined as the complete disappearance of the lesion lasting for more than 4 weeks. Partial response (PR) was defined as a decreased lesion size by more than $50 \%$ that remains stable for 4 weeks. Stable disease (SD) was defined as a tumor size change of less than $25 \%$ increase or less than $50 \%$ decrease. The sum of $\mathrm{CR}$ and $\mathrm{PR}$ was defined as response rate. Local control after ${ }^{125} \mathrm{I}$ brachytherapy was defined as lack of tumor progression either in or adjacent to the implanted site. For the calculation of overall survival, deaths due to any reason were scored as events. Pain intensity was measured and graded according to the Numerical Rating Scale (NRS) of the Adult Cancer Pain Clinical Practice Guidelines [17]: 0 indicated no pain, $1-3$ indicated mild pain, 4-6 indicated moderate pain, and 7-10 indicated severe pain.

\section{Statistical Analysis}

Local control rates and overall survival (OS) were the primary endpoints of this study. Data were calculated using the Kaplan-Meier method in SPSS 13.0 software (SPSS, Chicago, USA).

\section{Results}

\section{Pain Relief and Response to Treatment}

Before ${ }^{125} \mathrm{I}$ seed implantation, from the 36 patients, nine experienced severe pain (NRS 7-10), 19 patients had moderate pain (NRS 4-6), and eight had mild pain (NRS 1-3). Most patients reported pain relief 2-8 days after treatment. Four patients showed no change after implantation: two patients still felt moderate pain, one patient felt mild pain and one patient felt severe pain (Table 3). Pain relief response rate was $88.9 \%$.

Tumor response was evaluated after repeated clinical examination, ultrasound, and $\mathrm{CT}$ examination. The results revealed that eight patients achieved complete response (CR), twenty-two patients achieved partial response (PR), five patients showed stable disease (SD), and progressive disease (PD) was present in one case. The overall response rate $(\mathrm{CR}+\mathrm{PR})$ in this period was $83.3 \%$. 
Table 3 change of pain score

\begin{tabular}{lllll}
\hline & No pain $(\%)$ & Mild pain $(\%)$ & Moderate pain $(\%)$ & Severe pain $(\%)$ \\
\hline Pre-treament & $0(0 / 36)$ & $22.2(8 / 36)$ & $56.8(19 / 36)$ & $25(9 / 36)$ \\
Post-teament & $33.3(12 / 36)$ & $47.2(17 / 36)$ & $16.7(6 / 36)$ & $2.8(1 / 36)$ \\
\hline
\end{tabular}

Data are presented as \%(cases)

\section{Local Control and Survival}

The follow-up period ranged from 6 to 53 months. A locoregional failure was defined as tumor progression either in or adjacent to the implanted volume. The median local control was 28 months (95\% CI 16.2-39.8 months). The percentage of patients who showed 6-month, 1-year, 2-year, and 3 -year local control was $97.2 \%, 77.8 \%, 52.8 \%$, and $33.3 \%$, respectively (Fig. 1).

Twenty-one patients died of distant metastasis. Fifteen patients survived during this follow-up period. Median survival time for all patients was 48 months (95\% CI 40.955.1 months); 1-year, 2-year, 3-year, and 4-year survival rates were $97.2 \%, 80.6 \%, 63.9 \%$, and $46.5 \%$, respectively (Fig. 2).

\section{Complications and Adverse Reactions}

During the follow-up period, no serious complications due to ${ }^{125}$ I seeds were detected. Two patients suffered from localized skin erythema 8 days post implantation, but it disappeared after topical treatment. No ${ }^{125}$ I seeds migrated to other distant tissues or organs. Twenty-eight patients under combination chemotherapy showed varying degrees of leucopenia, liver or renal function impairment. These complications were reversed through symptomatic treatment.

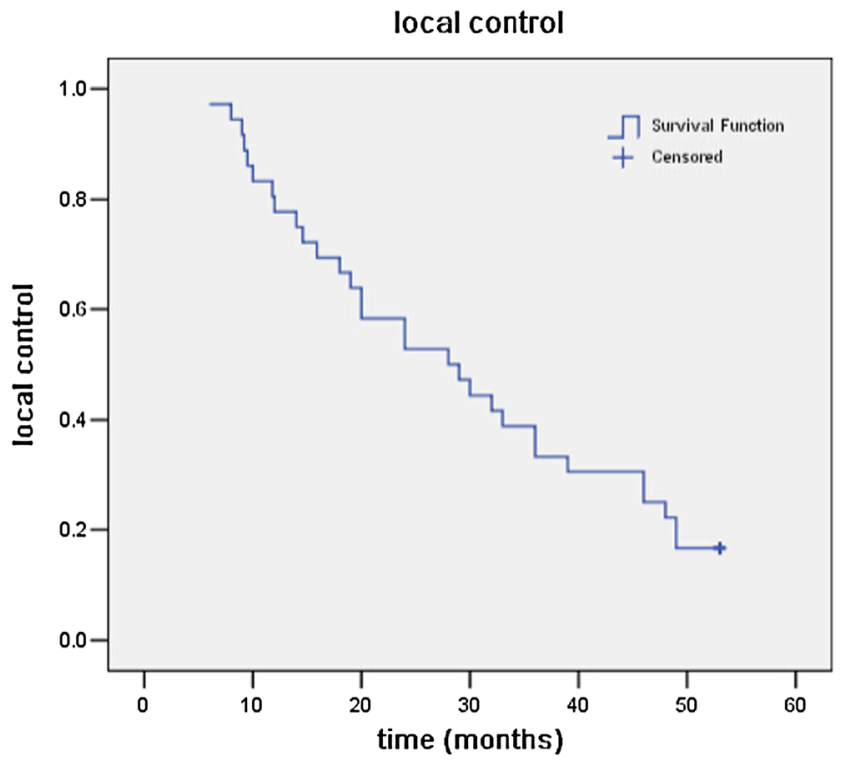

Fig. 1 Local control for all patients after iodine-125 seed implantation therapy

\section{Discussion}

LRBC represents a therapeutic challenge, especially the cases previously treated with multi-modality therapy. Nevertheless, LRBC alone may not always herald a fatal outcome and should not be a predicament for palliative treatment. On the contrary, LRBC treatment strategies should be tolerable, effective, and with long-lasting local control to improve the quality of life. The treatment strategies depend on the type of prior surgery, with or without adjuvant RT [18]. Other variables that could influence the outcome should also be considered: the size and the type of lesion (superficial diffuse or nodular), presence or absence of coexisting metastatic disease, time interval between the first treatment and retreatment, former chemotherapy or endocrine therapy, and other key factors.

Nowadays, surgery, chemotherapy, and EBRT are commonly applied as palliative treatments in LRBC. Local radical resection alone can only be offered to one-third of the patients with recurrence $[19,20]$, and they achieved a 5-year diseasefree survival rate of $45.5 \%$ [21]. Although local radical surgery is usually recommended, this may not be feasible in sizeable tumors [22]. Chemotherapy for LRBC has also been explored, but it was not found to be very effective [21]. Repeated EBRT has also limited efficacy in these patients. Reirradiation (ReRT) with full EBRT doses could increase the occurrence of both acute and late morbidities, because

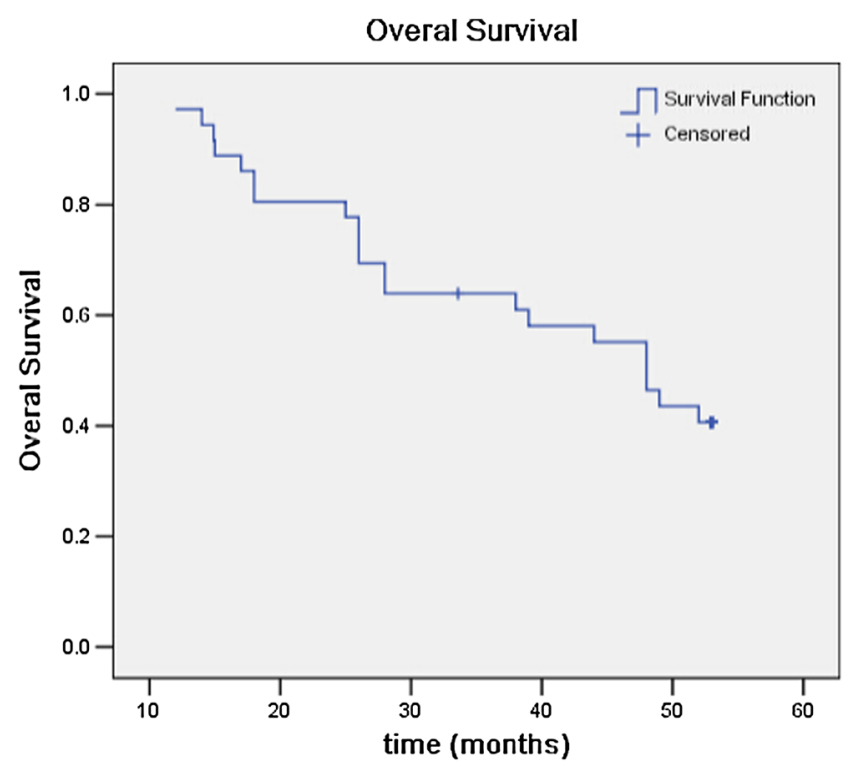

Fig. 2 Overall survival for all patients after iodine-125 seed implantation therapy 
most patients with LRBC have received prior RT, thus ReRT could represent a risk of exceeding radiation tolerance limits. For tumors $>5 \mathrm{~cm}$, it is difficult to deliver a large enough dose with EBRT, because RT should avoid damaging the adjacent normal tissues such as the lung, spinal cord, and heart [23]. A study reported that for tumors $>5 \mathrm{~cm}$, the effective EBRT dose is always greater than 100 Gy [24].

The ${ }^{125}$ I seed implantation method has received more and more attention in view of slight injury to normal tissues. This therapy was initially used for the treatment of prostate cancer and has also been investigated in the treatment of other solid tumors in recent decades [7-10]. There are studies documenting its efficacy in terms of local tumor control [25-27].

In the present retrospective study, we demonstrated that patients with LRBC could benefit from ${ }^{125}$ I seed implantation, given that the method yielded acceptable median survival and progression-free survival times, without any serious complications.

Our results indicate that ${ }^{125}$ I seed implantation exerts the following potential advantages: (1) it has a relatively long half-life, thus extending the radiation effects on the tumor cells; (2) ${ }^{125} \mathrm{I}$ seed implantation is not affected by respiratory movements which decrease the therapeutic volume in EBRT; (3) for tumors $>5 \mathrm{~cm}$, it delivers a large enough dose of localized radiation due to particle density, leading to tumor shrinkage without serious damage to the adjacent normal tissues; (4) ${ }^{125}$ I seeds have a low risk of side effects [28] and do not cause serious complications such as lymphedema, radiation pneumonitis, and pericarditis; (5) under local anesthesia, the ${ }^{125}$ I seed implantation technique can be performed easily, and the treatment time is shorter; (6) ${ }^{125}$ I seed radiation dose decreases with increasing distance, therefore reducing the damage to the adjacent normal tissues [29-31]. Based on our current results, we conclude that ${ }^{125}$ I seed implantation is clinically effective for locoregionally recurrent and unresectable breast cancers, reducing pain and remarkably improving the patients' quality of life.

However, our study also has some limitations, due to the retrospective nature of this analysis. First, some patients had to be excluded because their treatment post recurrence course or outcome could not be analyzed; second, we did not have a control group treated with other therapies and with similarly localized tumor recurrence. Finally, our study was based on a small number of patients and a short follow-up time. Therefore, large and well-designed prospective trials are still needed to refine the patient selection criteria, the optimal ${ }^{125} \mathrm{I}$ seed implantation dose, re-implantation interval time, and ideal treatment parameters.

\section{Conclusions}

Our results demonstrate that ${ }^{125}$ I seed implantation could be considered as a feasible and promising minimally invasive therapy for locoregionally recurrent and unresectable breast carcinoma, since it appears to be safe, inducing small damage, few adverse effects, slight pain, and can be performed in a short amount of time.

Acknowledgements The authors wish to thank Chiliang Chen of Iowa State University for critical reading of our manuscript. Thank Ying Cui and Jun-ming Huang for having been giving us so many valuable suggestions. At last, I wish to thank Xiao Zhu for making the references unavailable at our hospital library.

Funding This research was supported by Guangxi Scientific Research and Technology Development Projects (project number: 1,355,005-312), Guangxi Colleges and Universities Science and Technology Research Projects (project number: KY2015LX054), Humanities base project of Guangxi Medical University (project number: 2016RWB04) and Innovation Project of Guangxi Graduate Education (project number: YCBZ2017041).

\section{Compliance with Ethical Standards}

Conflict of Interest The authors of the article declare that they have no competing interests.

Open Access This article is distributed under the terms of the Creative Commons Attribution 4.0 International License (http:// creativecommons.org/licenses/by/4.0/), which permits unrestricted use, distribution, and reproduction in any medium, provided you give appropriate credit to the original author(s) and the source, provide a link to the Creative Commons license, and indicate if changes were made.

\section{References}

1. Khakpour N, Lucci A (2005) Repeat breast-conserving surgery for in-breast local breast carcinoma recurrence: the potential role of partial breast irradiation. Women's Oncol Rev 5:75-76

2. Fisher B, Anderson S, Bryant J, Margolese RG, Deutsch M et al (2002) Twenty-year follow-up of a randomized trial comparing total mastectomy, lumpectomy, and lumpectomy plus irradiation for the treatment of invasive breast cancer. N Engl J Med 347:1233-1241

3. Buchholz T (2011) 3-29 Locoregional radiation therapy in patients with high-risk breast cancer receiving adjuvant chemotherapy: 20year results of the British Columbia randomized trial. Breast Dis Year B Q 5:269-271

4. Cheng L, Swartz MD, Zhao H, Kapadia AS, Lai D et al (2012) Hazard of recurrence among women after primary breast cancer treatment-a 10-year follow-up using data from SEER-Medicare. Cancer Epidemiol Biomarkers Prev 21:800-809

5. Siglin J, Champ CE, Vakhnenko Y, Anne PR, Simone NL (2012) Radiation therapy for locally recurrent breast cancer. Int J Breast Cancer 2012:571946-571946

6. Rauschecker H, Clarke M, Gatzemeier W, Recht A (2001) Systemic therapy for treating locoregional recurrence in women with breast cancer. Cochrane Database Syst Rev (4):CD002195. https://doi.org/10.1002/14651858.CD002195

7. Li Y-f, Z-q L, Y-s Z, L-m D, C-y W et al (2016) Implantation of radioactive 125 I seeds improves the prognosis of locally advanced pancreatic cancer patients: a retrospective study. J Huazhong Univ Sci Technol Med Sci 36:205-210

8. Niu L, Luo X, Zeng J, Kong X, Fang G et al (2016) Cryoablation combined with Iodine-125 implantation in the treatment of cardiac 
metastasis from alveolar soft part sarcoma: a case report. Biomedicine Hub 1:4-4

9. Duan C, Zheng L, Jin M, Zhang D, Zhao W et al (2016) Combined chemotherapy and 125 I-particle implantation for treatment of children with head and neck soft tissue sarcomas improve the shortterm efficacy: Beijing Children's hospital experience over 2 years. Int J Clin Exp Med 9:11901-11906

10. Mahmood U, Blanchard P, Pugh TJ, Swanson D, Graber W et al (2016) Prospective cohort of permanent seed implantation prostate brachytherapy for intermediate risk prostate cancer: analysis of patient satisfaction and interference with daily activities between 125-iodine, 103palladium and 131-cesium. Brachytherapy 15:S201-S202

11. Wang JJ, Yuan HS, Li JN, Jiang YL, Tian SQ et al (2010) CTguided radioactive seed implantation for recurrent rectal carcinoma after multiple therapy. Med Oncol 27:421-429

12. Cox CE, Furman B, Stowell N, Ebert M, Clark J et al (2003) Radioactive seed localization breast biopsy and lumpectomy: can specimen radiographs be eliminated? Ann Surg Oncol 10:1039-1047

13. van Riet YE, Maaskant AJ, Creemers GJ, van Warmerdam LJ, Jansen FH et al (2010) Identification of residual breast tumour localization after neo-adjuvant chemotherapy using a radioactive 125 iodine seed. Eur J Surg Oncol 36:164-169

14. Mullins JP, Grams MP, Herman MG, Brinkmann DH, Antolak JA (2016) Treatment planning for metals using an extended CT number scale. J Appl Clin Med Phys 17(6):179-188

15. Alnaghy S, Loo K, Cutajar D, Jalayer M, Tenconi C et al (2016) BrachyView: multiple seed position reconstruction and comparison with CT post-implant dosimetry. J Instrum 11:P05002

16. Miller A, Hoogstraten B, Staquet M, Winkler A (1981) Reporting results of cancer treatment. Cancer 47:207-214

17. pain Coc (1986) Descriptions of chronic pain syndromes and definitions of pain terms: prepared by the International Association for the Study of Pain, subcommittee on taxonomy. Pain Suppl 3:S1-226

18. Cardoso F, Harbeck N, Fallowfield L, Kyriakides S, Senkus E, et al. (2012) Locally recurrent or metastatic breast cancer: ESMO clinical practice guidelines for diagnosis, treatment and follow-up. Annals of oncology 23: vii11-vii19

19. Dahlstrøm KK, Andersson AP, Andersen M, Krag C (1993) Wide local excision of recurrent breast cancer in the thoracic wall. Cancer 72:774-777
20. Bethke KP (1996) Breast conservation: predictors and treatment of local recurrence. Wiley online Library pp. 332-338

21. Petrella F, Radice D, Borri A, Galetta D, Gasparri R, Casiraghi M et al (2014) Chest wall resection and reconstruction for locally recurrent breast cancer: from technical aspects to biological assessment. Surg J R Coll Surg Edinb Irel 14(1):26

22. Salvadori B, Marubini E, Miceli R, Conti A, Cusumano F et al (1999) Reoperation for locally recurrent breast cancer in patients previously treated with conservative surgery. Br J Surg 86:84-87

23. Li W, Guan J, Yang L, Zheng X, Yu Y et al (2015) Iodine-125 brachytherapy improved overall survival of patients with inoperable stage III/IV non-small cell lung cancer versus the conventional radiotherapy. Med Oncol 32:1-8

24. Reveiz L, Rueda JR, Cardona AF (2012) Palliative endobronchial brachytherapy for non-small cell lung cancer. The Cochrane Library

25. Shi L, Li X, Pei H, Zhao J, Qiang W et al (2016) Phase II study of computed tomography-guided 125 I-seed implantation plus chemotherapy for locally recurrent rectal cancer. Radiother Oncol 118:375-381

26. Brahimaj B, Lamba M, Breneman JC, Warnick RE (2016) Iodine125 seed migration within brain parenchyma after brachytherapy for brain metastasis: case report. J Neurosurg 125(5):1167-1170

27. Welch H, Gorski D, Albertsen P, Andriole G, Crawford E et al (2016) Trends in metastatic breast and prostate cancer. N Engl J Med 2016:594-596

28. Dahiya M (2016) Brachytherapy: A review. J Crit Rev 3:6-10

29. Wierzbicka M, Bartochowska A, Strnad V, Strojan P, Mendenhall WM et al (2016) The role of brachytherapy in the treatment of squamous cell carcinoma of the head and neck. Eur Arch Otorhinolaryngol 273:269-276

30. Rasmusson E, Gunnlaugsson A, Kjellén E, Nilsson P, Einarsdottir M, Wieslander E, Fransson P, Ahlgen G, Blom R (2016) Low-dose rate brachytherapy with I-125 seeds has an excellent 5-year outcome with few side effects in patients with low-risk prostate cancer. Acta Oncol 55(8):1016-1021

31. Huo X, Wang H, Yang J, Li X, Yan W et al (2016) Effectiveness and safety of CT-guided 125 I seed brachytherapy for postoperative locoregional recurrence in patients with non-small cell lung cancer. Brachytherapy 15:370-380 